\title{
Transatlantica
}

Revue d'études américaines. American Studies Journal

\section{L'arc-en-ciel et le mouvement gai et lesbien}

Réfraction, dispersion et instrumentalisation des identités collectives

\section{Guillaume Marche}

\section{(2) OpenEdition}

Journals

Édition électronique

URL : http://journals.openedition.org/transatlantica/321

DOI : 10.4000/transatlantica.321

ISSN : $1765-2766$

Éditeur

AFEA

Référence électronique

Guillaume Marche, «L'arc-en-ciel et le mouvement gai et lesbien », Transatlantica [En ligne], 1 | 2005,

mis en ligne le 24 mars 2006, consulté le 29 avril 2021. URL : http://journals.openedition.org/

transatlantica/321 ; DOI : https://doi.org/10.4000/transatlantica.321

Ce document a été généré automatiquement le 29 avril 2021.

\section{(c) ()}

Transatlantica - Revue d'études américaines est mis à disposition selon les termes de la licence Creative Commons Attribution - Pas d'Utilisation Commerciale - Pas de Modification 4.0 International. 


\title{
L'arc-en-ciel et le mouvement gai et lesbien
}

\author{
Réfraction, dispersion et instrumentalisation des identités collectives
}

\author{
Guillaume Marche
}

1 L'arc-en-ciel est, aux Etats-Unis, un symbole à présent très répandu et quasi universellement connu de la communauté homosexuelle ${ }^{1}$. Cet emblème d'une homosexualité assumée et revendiquée est visible, généralement sous forme de drapeaux ou d'autocollants, sur la vitrine de commerces tenus par des personnes homosexuelles ou destinés à cette clientèle, sur le pare-choc d'automobiles appartenant à des gais ou à des lesbiennes désireux de signifier jusque sur la route leur appartenance à cette communauté, et même parfois à la fenêtre du domicile privé de personnes souhaitant afficher au vu et au su de tous les passants ce qu'il est convenu d'appeler leur orientation ou leur préférence sexuelle ${ }^{2}$, comme il est fréquent, aux Etats-Unis, de le faire de ses opinions politiques avec un drapeau portant le nom d'un parti ou d'un candidat, lors d'une élection.

2 Signifiant quasi immédiat d'une identité dont, au contraire, les contours objectifs sont flous et l'expérience subjective plurivoque, l'arc-en-ciel gai et lesbien ne laisse pas de soulever des questions profondes en termes de représentation, d'expression et de mobilisation identitaires. Ce symbole juxtaposant plusieurs bandes unies de couleurs différentes dans l'ordre où elles apparaissent dans le spectre lumineux n'est, en effet, qu'une assez lointaine évocation du phénomène de réfraction de la lumière blanche. L'agrégation des personnes homosexuelles sous une même bannière portant ce motif accomplit-elle une déformation et une simplification aussi considérables de l'identité de celles et ceux qu'il représente? En d'autres termes, le motif de l'arc-en-ciel peut-il être considéré, par métonymie, comme l'illustration voire le symptôme d'une dynamique réductrice qui serait à l'œuvre dans la représentation collective de l'identité subjective des gais et des lesbiennes dans l'espace public? Etudier la place du symbole de l'arc-en-ciel dans la mobilisation identitaire gaie et lesbienne aux Etats-Unis revient ainsi à s'interroger sur les modalités et la signification de la mise en rapport du privé au public et du sexuel au politique. 
3 Les origines de l'utilisation de l'arc-en-ciel comme symbole de l'identité gaie et lesbienne permettent de comprendre les termes dans lesquels se pose la politisation de l'homosexualité aux Etats-Unis, depuis les années 1970. Mais le devenir de ce symbole au-delà des premières étapes du mouvement gai et lesbien contemporain conduit à identifier des dynamiques d'institutionnalisation et de marchandisation, qui détournent l'arc-en-ciel de sa signification politique originelle. Partant, il importe d'examiner ces dynamiques, afin de déterminer si elles suivent des logiques différentes ou si elles relèvent, au contraire, d'une même instrumentalisation de l'identité collective gaie et lesbienne. La réponse apportée à cette question conduit in fine à discerner les voies par lesquelles la mobilisation identitaire gaie et lesbienne peut surmonter les impasses politiques dont certains usages de la figure de l'arc-en-ciel sont le symptôme. Nous verrons à ce titre que le motif de l'arc-en-ciel tel qu'il est communément représenté, d'une part, et l'arc-en-ciel comme spectre lumineux continu, d'autre part, correspondent à deux pôles permettant d'appréhender deux paradigmes concurrents dans la manière dont l'identité gaie et lesbienne est mobilisée dans l'espace public aux Etats-Unis.

\section{L'arc-en-ciel : origine d'un symbole}

4 L'arc-en-ciel est devenu le symbole de la communauté homosexuelle à partir de la fin des années 1970, aux Etats-Unis. Il fut créé en 1978 par Gilbert Baker, un artiste de San Francisco, en réponse à la demande du milieu activiste qui souhaitait se doter d'un symbole visuel d'identification collective. L'idée de ce thème lui vint d'un motif traditionnellement associé à diverses causes contestataires pendant les années 1960 : le drapeau créé par Baker se voulait un symbole de lutte, signification déjà associée au motif de l'arc-en-ciel depuis que le mouvement des coopératives alternatives au capitalisme en avait fait son symbole dans les premières décennies du XX $\mathrm{XX}^{\mathrm{e}}$ siècle $^{3}$. Il se composait, à l'origine, de huit bandes dont les couleurs devaient représenter divers aspects de l'expérience minoritaire des personnes homosexuelles américaines et de leurs aspirations: sexualité, vie, sérénité et accord avec la nature, harmonie et équilibre, pour n'en citer que quelques uns. La généralisation d'un drapeau à six bandes est, en réalité, le fruit du hasard. Le prototype présenté lors de la marche des fiertés homosexuelles de San Francisco en 1978 connut, en effet, un succès tel que ses créateurs voulurent le fabriquer en plus grand nombre, pour en faire un emblème facilement disponible, le mot d'ordre de l'époque étant de "sortir du placard", c'est-à-dire de contribuer à la visibilité des personnes homosexuelles, alors considérée comme le premier remède à leur oppression. Mais, le rose et le turquoise n'étant pas des couleurs de tissu facilement disponibles sur le marché, le nombre de bandes colorées fut réduit à six; et c'est sous cette forme que le drapeau arc-en-ciel est commercialisé depuis les années $1970^{4}$.

5 Ce drapeau a donc vu le jour dans le cadre de la proclamation publique de l'homosexualité, c'est-à-dire de la revendication politique d'une identité on ne peut plus privée et ce, dans un contexte historique où, après bientôt dix ans d'effervescence militante, les personnes homosexuelles étaient soumises à de violentes attaques allant de la campagne d'Anita Bryant, destinée à remettre en cause leur égalité de droits dans le comté de Miami, en Floride, à l'initiative de John Briggs pour leur interdire la profession d'enseignant, en Californie ${ }^{5}$. Dès lors, "sortir du placard» et assumer, individuellement ou collectivement, son homosexualité constituait un défi effectif aux discours stigmatisants dont elle faisait l'objet : en se désignant soi-même comme gai ${ }^{6}$, 
l'on cessait justement d'être l'objet d'une désignation et l'on en désamorçait le caractère stigmatisant, pour transmuer l'opprobre et la honte en capacité d'être le sujet d'une action sur sa propre expérience ${ }^{7}$. Ainsi, à la fin des années 1970, cette démarche d'auto-désignation était synonyme de revendication d'appartenance à un mouvement social et le drapeau arc-en-ciel constituait l'emblème collectif d'une identité implicitement politisée.

6 L'émergence de l'arc-en-ciel comme signe de ralliement homosexuel est également contemporain de la constitution d'enclaves gaies et lesbiennes dans les grandes villes américaines ${ }^{8}$. Celles-ci résultèrent d'une démarche volontaire visant à atteindre deux objectifs complémentaires, dont le premier était de permettre aux gais et aux lesbiennes de vivre leur homosexualité au grand jour dans des conditions confortables, grâce à un ensemble de commerces et de services à même de répondre à leurs besoins spécifiques, ou bien de pourvoir aux besoins de leur vie ordinaire sans exercer à leur encontre de discrimination'. Notons que ce communautarisme, d'inspiration somme toute capitaliste, ainsi que la nature des commerces et services concernés, dont beaucoup - tels les sex-shops ou les bains - répondaient à une demande d'ordre sexuel, reflétaient surtout un communautarisme d'hommes homosexuels ${ }^{10}$. De nombreuses lesbiennes s'identifiaient plus volontiers au féminisme lesbien, qui inspirait alors un communautarisme lesbien conçu comme une stratégie permettant aux femmes de résister en édifiant des alternatives au modèle dominant de société, mû par des valeurs toutes masculines d'appropriation et de domination, dont la sexualité masculine était considérée comme un emblème ${ }^{11}$.

7 Il n'en demeure pas moins que les ponts n'étaient pas rompus entre les homosexuels de l'un et l'autre sexe, puisque toutes les lesbiennes, loin s'en faut, ne s'identifièrent pas à la séparation d'avec les hommes homosexuels qu'induisait le féminisme lesbien ${ }^{12}$. Le projet de constitution d'enclaves urbaines avait, au reste, vocation à représenter les personnes homosexuelles indépendamment de leur sexe. Le second objectif était, en effet, de permettre à l'ensemble de la population homosexuelle d'accéder à une plus grande visibilité dans le domaine public, notamment en devenant un groupe numériquement significatif en termes de représentation électorale au plan municipal. A San Francisco, Harvey Milk incarna le succès de cette stratégie d'appropriation de l'espace urbain en se faisant élire en novembre 1977 au conseil municipal comme représentant de la circonscription correspondant au quartier du Castro, désormais identifié à la communauté gaie et lesbienne ${ }^{13}$.

8 Il n'est donc rien d'étonnant à ce que ce soit justement au cœur de ce quartier qu'en 1985, une place ait été nommée en sa mémoire, après son assassinat en novembre 1978, et qu'elle soit parée depuis 1997, c'est-à-dire depuis le vingtième anniversaire de son élection, et non de sa mort, d'un immense drapeau arc-en-ciel ${ }^{14}$. Ce drapeau demeure l'emblème d'une appropriation de l'espace public à des fins d'affirmation collective, lesquelles se manifestent notamment lors des défilés annuels des fiertés homosexuelles commémorant les émeutes de Stonewall qui, à New York, en juin 1969, marquèrent un tournant dans la mobilisation collective homosexuelle en précipitant la formation du mouvement gai de libération ${ }^{15}$. Ce mouvement se démarque des mobilisations homosexuelles antérieures dans la mesure où il est axé sur la revendication conjointe d'une identité offensive ne reniant rien de sa déviance, et d'une citoyenneté de plein droit, revendication visant in fine à transformer la société dans son ensemble ${ }^{16}$. Les défilés des fiertés homosexuelles furent conçus pour remettre en scène, dans le topos 
urbain, cette dynamique par laquelle les gais et les lesbiennes investissent temporairement un espace public qui n'est pas spécifiquement désigné comme le leur. La rue des enclaves urbaines gaies et lesbiennes est, quant à elle, le théâtre permanent d'activités politiques, notamment parce qu'elle permet l'affichage d'informations ou de campagnes concernant la communauté. L'adoption de l'arc-en-ciel par cette communauté est donc indissociable d'une dynamique de revendication d'une identité collective qui se voulait tout à la fois porteuse de bien-être pour les personnes homosexuelles elles-mêmes et facteur de progrès pour la société dans son ensemble ${ }^{17}$.

\section{Marchandisation et institutionnalisation}

9 La signification militante de l'arc-en-ciel est néanmoins soumise à deux remises en cause, dont la première a trait aux deux objectifs sur lesquels reposent les enclaves urbaines homosexuelles. Une fois acquise une situation dans laquelle le quartier constitue effectivement un havre où nul n'est entravé dans le bon déroulement de sa vie quotidienne au motif de son homosexualité, l'objectif de "confort» exposé plus haut se mue en consumérisme. Parallèlement, l'objectif politique de "visibilité " connut, au cours des années 1970, un succès tel que l'homosexualité put bientôt bénéficier d'un plus haut degré d'acceptation de la part de personnes que les gais et les lesbiennes des grands centres urbains étaient amenés à côtoyer, si bien que la nécessité d'un engagement politique pouvait se faire moins ressentir ${ }^{18}$.

Or, l'usage de l'arc-en-ciel témoigne de cette mutation : de symbole militant il est, dans une large mesure, devenu symbole marchand, motif multicolore que l'on retrouve sur toute une gamme de produits de consommation, du t-shirt au bijou et du porte-clé au distributeur de savon liquide pour la salle de bains ${ }^{19}$. Outre le fait que les articles de maison ainsi vendus sont destinés à agrémenter des espaces privés, même dans le cas d'objets que l'on arbore comme signe d'appartenance, il y a une différence de nature entre un drapeau que l'on brandit pour accompagner un geste d'ordre politique et le motif ornant une marchandise dont l'acquisition se suffit à elle-même. De même, dans l'espace public des quartiers gais et lesbiens, l'activité purement commerciale de la rue prend souvent le pas sur l'engagement civique, comme l'illustre l'exemple même du quartier san-franciscain du Castro, qui fut longtemps un lieu tout à la fois de consommation, de solidarité et de contestation. En 1998, néanmoins, les commerçants de Castro Street et de la $18^{\mathrm{e}}$ rue firent interdire la mendicité sur le trottoir et l'affichage sur les lampadaires ${ }^{20}$. Le quartier était, en effet, devenu une attraction touristique, au même titre que le quartier chinois de Chinatown ou le quartier italien de North Beach, ce dont les commerçants souhaitaient tirer pleinement parti sur le plan commercial, privilégiant l'attractivité vis-à-vis de clients potentiellement extérieurs à la communauté, au détriment de la solidarité à l'égard de personnes ou d'organisations $a$ priori parties prenantes au projet initial de cette enclave urbaine homosexuelle, lequel reposait justement sur l'idée qu'il n'y ait pas de solution de continuité entre objectifs économiques et politiques.

11 Dans les faits, c'est plus largement l'identité collective gaie et lesbienne elle-même qui est soumise au processus de marchandisation. Ainsi, en 1997, le défilé de la Gay Pride de Boston fut suivi d'une gigantesque soirée dansante intitulée "Revolution». Le contraste entre l'apparence politique que lui conférait ce nom et la nature entièrement ludique et hédoniste de la manifestation est d'autant plus frappant que la participation était, comme on peut s'y attendre, soumise à un prix d'entrée non négligeable ${ }^{21}$. Les organisateurs cherchaient donc à provoquer une envie de défoulement et de 
consommation, en la faisant passer pour un engagement dans le combat pour l'égalité et ce, principalement dans le but de réaliser un profit. Pour caricatural qu'il soit, cet exemple n'illustre pas moins la manière dont les signifiants identitaires gais et lesbiens sont fréquemment détournés afin de produire ce qu'il faut bien appeler un simulacre de mouvement social.

Cette marchandisation correspond donc à une forme d'instrumentalisation de l'identité collective, dont l'évolution de la signification effective de l'arc-en-ciel est emblématique. Mais cette identité est soumise à une autre forme d'instrumentalisation, corollaire de la première, dont la figure de l'arc-en-ciel permet également de rendre compte. La marchandisation a, en effet, pris corps à mesure que le communautarisme homosexuel prenait le pas sur la lutte pour la libération gaie ${ }^{22}$, de sorte que l'identité collective mobilisée dans le champ du mouvement politique s'est fortement homogénéisée. Dans une Amérique des années 1970 encline au renouveau ethnique, l'arc-en-ciel des origines était l'emblème d'une mobilisation gaie et lesbienne assumant la diversité de ses composantes ${ }^{23}$. C'est ce qu'illustre, par exemple, le refus de la Gay Activist Alliance de mener avec la municipalité de New York, en 1971, des négociations en vue d'interdire la discrimination en fonction de l'orientation sexuelle tant que les personnes travesties formant une part importante de sa base ne seraient pas autorisées à y participer ${ }^{24}$. Aux yeux de ces militants, en réalité, il n'y avait pas une différence de nature, mais de style entre l'identité politique des personnes homosexuelles travesties et celle des autres, la transgression des frontières de genre étant conçue - au lendemain d'émeutes dont un groupe d'hommes travestis africains américains et portoricains avaient été les déclencheurs - comme une modalité subjective parmi d'autres d'une identité collective qui revendiquait sa diversité.

Dès la fin de la décennie, en revanche, la responsabilité de l'action collective était de plus en plus largement déléguée à des leaders, qui prirent en charge l'initiative d'un mouvement désormais communautaire. Ils firent ainsi, assez logiquement, appel à une identité collective gaie et lesbienne définie sur le modèle des identités ethniques, afin de profiter des possibilités de reconnaissance institutionnelle offertes à ces dernières ${ }^{25}$. Cette "ethnicisation" reposait sur une certaine essentialisation de l'homosexualité - présentée comme une composante primordiale de l'individualité de chaque personne homosexuelle-et mettait par conséquent l'accent sur la communauté d'expérience des membres du groupe minoritaire, donc sur son homogénéitée ${ }^{26}$, au point d'être perçue comme exclusive par les personnes homosexuelles non blanches ${ }^{27}$. L'ouverture institutionnelle faite aux homosexuels américains au début des années 1990, dans le contexte d'une visibilité accrue due au $\operatorname{sida}^{28}$ et de l'élection de Bill Clinton à la Maison Blanche, eut pour effet de renforcer ce processus d'homogénéisation, facteur d'intégration dans un système pluraliste de promotion d'intérêts collectifs spécifiques. De surcroît, le revers majeur subi par la cause des droits des gais et des lesbiennes avec l'arrêt Bowers v. Hardwick de la Cour Suprême - autorisant en 1986 l'État de Georgie à prohiber et réprimer des rapports sexuels homosexuels entre adultes consentants dans un espace privé - avait semblé donner raison aux tenants d'une stratégie d'intégration institutionnelle, qui recommandaient d'axer la défense des droits des gais et des lesbiennes sur une désexualisation de l'homosexualité et sur une définition de celle-ci en terme de statut (status) plutôt que de conduite (conduct) - c'est-à-dire de condition involontaire, 
permanente et irréversible, indépendamment des comportements spécifiques, notamment sexuels, qu'elle peut induire ${ }^{29}$.

Entre la fin des années 1980 et le début des années 1990, l'arc-en-ciel était ainsi devenu un emblème destiné à «vendre»-politiquement autant que commercialement - l'image d'une homosexualité somme toute sympathique et non menaçante ${ }^{30}$. Certes, les changements épidémiologiques de la transmission du VIH, mis en évidence au début des années 1990, conduisirent les organisations gaies et lesbiennes de lutte contre le sida à prendre en compte la diversité des homosexualités. Si l'on constatait de plus forts taux de transmission du VIH à l'occasion de rapports sexuels entre hommes issus des minorités ethniques que lors de rapports sexuels entre hommes blancs, c'était, en effet, que les premiers ne s'étaient pas sentis concernés par des messages de prévention qui avaient, en revanche, souvent sensibilisé les seconds ${ }^{31}$. Ainsi, le sida constituait un révélateur du fait qu'être homosexuel peut revêtir des significations différentes selon les individus, notamment en fonction de leur identité ethnique. Cette conception, défendue depuis longtemps, en particulier par des lesbiennes africaines américaines et latinas critiques vis-à-vis des tendances homogénéisantes du féminisme lesbien ${ }^{32}$, trouva alors un écho suffisant pour que les organisations gaies et lesbiennes - à l'instar des organisations de lutte contre le sida - se fissent fort de représenter les minorités ethniques au sein de la communauté. Mais, dans les faits, cet effort se traduisit souvent par de simples concessions faites à la diversité (tokenism), autrement dit par une sorte de parité de surface, dans laquelle les minoritaires sont autorisés à s'asseoir à la table mais pas à définir l'ordre du jour ${ }^{33}$.

15 Consentir une telle prise en compte a minima de la diversité signifie donc que l'on construit implicitement l'homosexualité comme une identité collective a priori blanche, mais au sein de laquelle existent des particularismes. Recourir à l'arc-en-ciel comme symbole de la communauté gaie et lesbienne revient dès lors à promouvoir l'image d'un groupe multicolore, qui laisse les différences s'épanouir en son sein. Ainsi, pour être multiculturelle, cette attitude mobilise une identité collective en réalité composite, dans laquelle se côtoient des éléments distincts les uns des autres. Mais, dans cet arc-en-ciel, chaque couleur n'est au fond qu'une variante d'un même modèle, si bien que le caractère offensif de l'arc-en-ciel protestataire des origines s'est dissipé pour laisser place à une homogénéité dans le motif, que tente de dissimuler la diversité affichée des couleurs. Le résultat en est que nombre de lesbiennes et de gais notamment issus des minorités ethniques se sont, depuis le milieu des années 1990, détournés d'un mouvement politique institutionnalisé qui instrumentalise l'identité collective $^{34}$. Par conséquent, l'institutionnalisation du mouvement gai et lesbien tend à homogénéiser la signification politique de l'homosexualité, ce qui conduit paradoxalement à un fort émiettement, voire à une fragmentation identitaire.

La figure de l'arc-en-ciel est donc soumise à deux modes de désamorçage distincts, mais qui suivent une même logique : que ce soit dans le champ politique ou dans le domaine commercial, l'homosexualité se vend mieux si elle est clairement identifiable à un item à la fois homogène et inoffensif. Ainsi, de même qu'existe dans la branche institutionnalisée du mouvement une volonté patente de banaliser l'homosexualité, certaines organisations gaies et lesbiennes - telles Strub Media Group ou Overlooked Opinions-mènent une politique active de commercialisation au sens propre de l'homosexualité, qui consiste littéralement à s'adresser aux investisseurs afin de leur vendre la communauté gaie et lesbienne comme un marché rentable ${ }^{35}$. Notons que cette 
stratégie met, dans les faits, l'accent sur le marché gai plutôt que lesbien, tant il est vrai que les hommes homosexuels, identifiables comme ayant des modes de consommation fortement axés sur la communauté gaie, sont à la fois plus nombreux et plus aisés que les femmes présentant un profil équivalent ${ }^{36}$. Toutefois, les années 1990 ont aussi vu les médias mettre en avant le personnage stéréotypé de la lesbienne élégante, censée représenter l'émergence d'une nouvelle génération de lesbiennes ayant troqué le souci, réputé dépassé et exagéré, de l'engagement politique contre celui de son apparence physique et de sa carrière ${ }^{37}$. La production d'un discours sur le créneau commercial homosexuel est ainsi, en réalité, moins déséquilibrée en termes de genre qu'il n'y paraitt à première vue.

17 Sur le plan politique comme sur le plan commercial, homogénéisation et désamorçage des différences deviennent donc, en somme, l'habillage obligatoire pour permettre l'accès à ce qu'il faut bien appeler le champ de la normalité et ce, en fonction d'une logique selon laquelle s'il y a un créneau, il convient de s'y conformer. Or, le répertoire disponible dans ce champ ne permet pas la représentation de l'arc-en-ciel comme un spectre continu: les matériaux nécessaires à une telle représentation n'y sont pas disponibles; partant, il est effectivement plus facile-en termes d'opportunité politique ou commerciale - d'adapter la représentation au vocabulaire disponible, que de chercher à faire évoluer ledit vocabulaire. Si arc-en-ciel il y a, c'est alors une représentation multicolore, plutôt que polychrome: des teintes variées sont, certes, présentées ; mais c'est dans un ensemble bariolé, chamarré, où prévaut la juxtaposition des couleurs, et non une sorte de fondu où elles se mêlent dans une certaine continuité.

La raison en est, en dernière analyse, que l'institutionnalisation et la commercialisation décrites ici consistent l'une comme l'autre en un processus dont l'initiative provient du haut et non de la base. Une comparaison avec ce qu'est, au sens propre, l'arc-en-ciel permet d'appréhender ce problème : le dictionnaire Robert définit l'arc-en-ciel comme un "phénomène météorologique et lumineux en forme d'arc, offrant les couleurs du prisme (violet, indigo, bleu, vert, jaune, orangé, rouge) et qui est produit par la réfraction et la dispersion des radiations colorées composant la lumière blanche par des gouttes d'eau $»^{38}$. En d'autres termes, l'arc-en-ciel est produit par des gouttelettes qui réfractent individuellement la lumière: là réside bien la différence entre le véritable arc-en-ciel et la représentation que l'on a l'habitude d'en voir, laquelle consiste en la juxtaposition, par la main de celui qui dessine, de six bandes de couleur distinctes.

Ce détour par la réfraction de la lumière met en évidence le fait que, dans les exemples qui précèdent, la problématique de la discontinuité est inséparable de celle de l'appropriation de l'identité collective gaie et lesbienne par une frange influente de la communauté. En effet, la prise en charge de l'initiative politique par un nombre restreint de leaders, initiée dès les années 1970, puis qui connut un recul face à l'urgente menace que représentait l'épidémie de sida au cours des années $1980^{39}$, s'intensifia au début des années 1990 avec le recours accru à des stratégies de lobbying et d'action en justice, plutôt que de protestation. Ainsi, de même que le commerce arc-en-ciel fait des personnes homosexuelles ses clients, il s'établit entre ces derniers et les leaders du mouvement institutionnalisé une relation de client à fournisseur d'un service ${ }^{40}$. Dans un cas comme dans l'autre, la mise en œuvre d'une identité collective correspondant à un arc-en-ciel formé de bandes juxtaposées n'est que la manifestation de la mainmise, largement consentie au moins de manière tacite, d'une fraction de la 
communauté sur un signifiant devenu commun à toutes les personnes homosexuelles. Partant, il convient de s'interroger à présent sur l'existence de démarches visant à redonner à l'arc-en-ciel gai et lesbien toute sa signification protestataire.

\section{Résistances et réappropriation}

Face au désamorçage de l'identité collective homosexuelle qui est largement mobilisée dans l'espace public, des initiatives se font jour qui visent à faire partir de la communauté gaie et lesbienne un mouvement contestataire. Tandis que la perspective d'une légalisation du mariage entre personnes de même sexe semble monopoliser le débat public sur l'homosexualité, l'antenne san-franciscaine de l'organisation Gay Shame revendique un discours politique plus offensif au nom de l'homosexualité. Par exemple, faisant allusion aux files de couples de même sexe, qui attendaient devant les bâtiments de l'administration municipale de San Francisco de pouvoir faire enregistrer leur procédure de mariage, après que le maire nouvellement élu, Gavin Newsom, eut pris l'initiative en février 2004 de procéder à de tels mariages, l'association déclare sans ambages :

Whatever happened to the time when being queer was an automatic challenge to the disgusting, oppressive, patriarchal institution of holy matrimony? Now, it seems that queers are so desperate to get their taste of straight privilege that they'll camp out in the rain with the hopes that the state will finally sanction their carnal coupling ${ }^{41}$.

Créée pour s'opposer à la banalisation (mainstreaming) de l'homosexualité en une caractéristique inoffensive, car inhérente à des sujets par ailleurs "normaux », cette organisation exprime ainsi un projet identitaire gai et lesbien qui, pour être nettement différentialiste - comme en témoignent l'usage de «queer » et la stigmatisation d'une institution que ses défenseurs considèrent comme le pilier de la civilisation occidentale -, n'est nullement séparatiste. Certes, les militants de Gay Shame ne souhaitent aucunement être intégrés à l'une des institutions maîtresses de la société dans laquelle ils vivent, mais c'est parce qu'ils jugent que c'est un système d'attribution de privilèges illégitimes qu'ils ont précisément à cœur de combattre ${ }^{42}$.

Cette volonté d'opposition contestataire s'exprime notamment à l'endroit de la Gay Pride, tenue, non sans quelque raison, pour responsable de la marchandisation de l'homosexualité : c'est précisément pour faire pièce au consumérisme béat de cette manifestation que les fondateurs de Gay Shame ont choisi ce nom. A San Francisco, les militants se joignent au cortège en portant des affichent clamant le slogan «queer mutiny-not consumer unity » et décernent, non sans humour, sur leur site Internet, des gratifications : les Gay Shame Awards. En 2003, les responsables de la Gay Pride se virent ainsi attribuer un prix de marchandisation de l'homme gai (« commodification of the gay male »), dont le trophée était un dollar arc-en-ciel (« a rainbow dollar ») : « for supporting local businesses by bringing all these queers to town, equating identity with corporate loyalty and robbing us of creativity and individuality in the process ${ }^{43}$. L'organisation visait, par ce message, à fustiger l'inanité de discours qui s'inspirent de l'idée reçue selon laquelle les hommes gais auraient un pouvoir d'achat supérieur à la moyenne ${ }^{44}$ pour encourager ces derniers à marquer leur approbation vis-à-vis d'entreprises réputées favorables aux droits des gais et des lesbiennes en leur accordant massivement leur clientèle ${ }^{45}$. A contrario, Gay Shame appelle de ses vœux la réactivation d'un militantisme pétri « de créativité et d'individualité », par opposition à une utilisation politique figée de l'identité homosexuelle, dont l'initiative vient du haut. 
C'est précisément ce à quoi se livre cette organisation en faisant entendre une voix dissidente au moyen d'initiatives aussi provocatrices qu'originales et facétieuses.

Exprimer dans l'espace public une voix différente qui parte de la base correspond bien au processus de réfraction décrit plus haut comme une alternative à la marchandisation et à l'institutionnalisation. C'est également ce à quoi s'emploie l'organisation san-franciscaine Lesbian and Gay Insurrection, dont l'acronyme LaGAI reprend lui aussi avec une dérision consommée la désignation identitaire "gay ». De manière significative, le journal de LaGAI s'intitule Ultra-Violet, en référence à une couleur communément associée à l'homosexualité mais poussée ici à son extrême : LaGAI se veut une organisation homosexuelle radicale; or, le titre de son journal désigne précisément l'une des couleurs de l'arc-en-ciel qui, pour n'être pas visible, n'en est pas moins une composante effective du spectre lumineux. Hostile, comme Gay Shame, à l'idée du mariage entre personnes de même sexe, cette organisation définit dans son journal les lesbiennes et les gais comme «the people who choose love, and sex, over societal acceptance, over physical security, over the almighty buck $»^{46}$. Le discours de LaGAI fait ici écho aux mots d'ordre du mouvement gai de libération des années 1970, selon lequel la famille nucléaire et le mariage sont des outils de répression de l'épanouissement individuel par le contrôle de la sexualité ${ }^{47}$. L'organisation articule son rejet de l'assimilationnisme matrimonial avec une critique de la marchandisation, posant la sexualité comme l'expression d'une subjectivité plutôt que d'un pouvoir d'achat.

Dans la rue comme dans les colonnes de son journal, LaGAI entend bien remettre en cause les stéréotypes positifs sur l'homosexualité, en particulier la représentation des gais et des lesbiennes comme des gens normaux et inoffensifs qui n'aspirent qu'à se marier. Durant l'été 1996, Ultra-Violet raillait ainsi les premiers frémissements du mouvement en faveur du mariage entre personnes de même sexe, en affirmant que les forces politiques conservatrices qui s'y opposaient tenaient surtout à interdire aux couples homosexuels la possibilité d'avoir accès au divorce, présenté comme corollaire du mariage. L'un de ces articles allait jusqu'à prêter au Président Clinton des propos fantaisistes dans lesquels il s'engageait à défendre l'institution du divorce contre les tentatives des homosexuels pour la pervertir ${ }^{48}$. Ce texte satirique visait à mettre en garde contre la stratégie de l'assimilationnisme en épinglant le Président des Etats-Unis qui, malgré ses déclarations favorables aux droits des gais et des lesbiennes, ne manifestait aucune volonté de s'opposer concrètement au Defense of Marriage Act ${ }^{49}$, qu'il allait de fait avaliser au début du mois d'août.

Ce même été 1996, une semaine avant la tenue officielle du défilé de la Gay Pride de San Francisco au mois de juin, LaGAI avait déjà organisé, en plein cœur du quartier gai et lesbien du Castro, un divorce collectif. Depuis une estrade, tous les volontaires pouvaient venir annoncer publiquement leur divorce d'avec la personne, l'institution ou l'objet de leur choix. "Some people who'd been together fifteen years came [on stage] and said yeah, let's divorce!» se rappelle avec délectation l'une des organisatrices ${ }^{50}$. D'autres divorçaient solennellement d'avec les Scouts ou diverses organisations religieuses connues pour leur homophobie, dans une atmosphère tout au rire et à la détente. Autour de l'estrade, étaient installés des stands : dans l'un, les passants pouvaient prendre part à un concours de scènes de ménage, où ils étaient invités à rivaliser d'ardeur pour briser de la vaisselle; un autre abritait l'agence de voyages Go Your Separate Ways, dans laquelle on faisait mine de proposer aux jeunes 
divorcés différentes formules de voyage de séparation; dans un autre encore, chacun pouvait se faire établir par les organisateurs un certificat de divorce ${ }^{51}$. Ce type d'intervention peut sembler vain en termes politiques, tant il s'apparente à une simple représentation interactive ou à du théâtre de rue, mais il permet, en réalité, à ceux qui se sentent marginalisés par la marchandisation et l'instrumentalisation étudiées ici d'exprimer dans l'espace public un point de vue dissident.

En l'espèce, à une période où la conquête du droit d'épouser une personne du même sexe semblait déjà de l'ordre du possible, si LaGAI avait demandé aux passants de se prononcer sur l'opportunité de chercher à obtenir ce droit, cette organisation aurait probablement recueilli des réponses majoritairement favorables, en vertu de la logique anti-discriminatoire selon laquelle les homosexuels doivent avoir droit à tout ce à quoi ont droit les hétérosexuels. Mais, en poussant par avance cette logique à l'extrême et en assimilant l'accès des couples de même sexe à l'institution du mariage à leur accès au divorce, les organisateurs du divorce collectif du 22 juin 1996 parvinrent à libérer une expression collective spontanée, car axée sur le divertissement. En somme, c'est justement parce que LaGAI aborda le thème du mariage sous l'angle du rire et de la parodie, que cette organisation put obtenir des participants à son divorce collectif qu'ils expriment une subjectivité authentique, plutôt qu'un discours politique contrôlé, étudié en fonction de l'image forcément positive qu'il est censé produire auprès des décideurs institutionnels, en vue d'obtenir une faveur spécifique de leur part. Cette organisation participe donc à la production d'un discours identitaire gai et lesbien qui s'affranchit de la contrainte de "vendre » une identité homosexuelle acceptable et suit, au contraire, le processus de réfraction défini plus haut.

Nonobstant la désexualisation de l'homosexualité qui prévaut dans les discours publics favorables aux droits des gais et des lesbiennes depuis le milieu des années 1980, en effet, la défense du droit au mariage n'empêche en rien, par exemple, la persistance d'arrestations pour outrage aux bonnes mœurs dans des lieux de rencontre fréquentés par des hommes homosexuels - que ces lieux soient ouverts, comme certains parcs ou aires de repos d'autoroute, ou fermés, comme un grand nombre de bars et de clubs - souvent à la suite de sollicitations de la part d'agents de police en civil ${ }^{52}$. Pour les défenseurs d'une homosexualité inoffensive, cautionner la stigmatisation des personnes homosexuelles les plus ouvertement déviantes en raison de leur comportement sexuel constitue, par là même, un gage de respectabilité. La mise en œuvre d'une identité homosexuelle fortement sexualisée contribue, au contraire, à contrecarrer conjointement la tendance assimilationniste à l'institutionnalisation du mouvement et la marchandisation de l'identité collective, par la revendication d'une sexualité non seulement audacieuse, mais axée sur la réciprocité et la gratuité. Les tenants d'une identité gaie et lesbienne fortement sexualisée revendiquent ainsi une homosexualité qui pose problème en prenant le parti de la déviance contre la normalité. Il s'agit pour eux, d'une part, de ne pas se désolidariser des personnes homosexuelles les plus stigmatisées, car, lorsque surviennent des épisodes de forte inquiétude pour la normalité sexuelle, les réactions d'intolérance ont tôt fait d'amalgamer tous ceux qui dévient de la norme hétérosexuelle, même les plus normaux en apparence : l'émergence du sida, au début des années 1980, valut ainsi la même stigmatisation à toutes les personnes homosexuelles, quelles qu'elles fussent, et notamment aux lesbiennes ${ }^{53}$. 

subjectivité des divers types d'acteurs que recouvre le terme homosexulité contre une acceptation a minima des personnes homosexuelles les plus présentables ${ }^{54}$. Cette mouvance s'emploie, en particulier, à contrecarrer les discours qui désexualisent l'homosexualité féminine, autre stéréotype prétendument positif qui présente le lesbianisme comme un type d'homosexualité acceptable, les lesbiennes étant, en tant que femmes, réputées moins portées sur une quête effrénée d'épanouissement sexuel que les hommes gais. Depuis les années 1980, de plus en plus de lesbiennes revendiquent, au contraire, une culture sexualisée du lesbianisme qui mette plus largement en cause le stéréotype de la sexualité féminine passive ${ }^{55}$. Critiquant la simple revendication de la protection de la vie privée, Amber Hollibaugh déclare :

The problem, of course, with a conservative defense is that you can't argue for the beauty or power or right of your own or others' sexuality when you are also arguing for sexual privacy-the don't ask, don't tell argument ${ }^{56}$.

Ce qui est en jeu, selon elle, c'est bien le choix entre la mise au silence de la sexualité lesbienne, qui est censée lui valoir une certaine tolérance, et sa reconnaissance, qui passe, elle, par le déploiement de la subjectivité des acteurs, et non par une certaine réserve sur la question sexuelle.

Si la notion de réfraction évoque, comme nous venons de le montrer, le principe d'une action collective dont l'initiative part de la base et reflète des subjectivités qui ne sont pas nécessairement conformes à ce que la société américaine peut d'ores et déjà intégrer, il en résulte justement un spectre diffus, où les couleurs sont indistinctes, c'est-à-dire qu'elles ne sont pas clairement identifiables à une valeur stable et unique. Face aux deux écueils que constituent une image monochrome et une représentation simplement multicolore de la communauté homosexuelle, d'aucuns s'emploient à conférer une véritable polychromie à l'arc-en-ciel gai et lesbien. Au milieu des années 1990, par exemple, tandis que deux cas de brutalité policière avaient touché, à peu de temps d'intervalle, d'une part, la communauté gaie et lesbienne et, d'autre part, la communauté africaine américaine de San Francisco ${ }^{57}$, des militants issus de ces deux groupes se rassemblèrent en un collectif informel, afin de faire valoir les droits des victimes dans ces deux affaires. Des militants issus d'autres minorités ethniques et de divers groupes stigmatisés, tels que les personnes prostituées, se joignirent bientôt à ce combat $^{58}$. Le collectif san-franciscain de lutte contre les brutalités policières rassemble donc des personnes d'identités très diverses, qui ne se considèrent aucunement comme semblables, mais se reconnaissent un intérêt commun. Ce collectif suscite donc des liens entre des groupes qui n'avaient pas l'habitude de coopérer. De manière encore plus significative, il crée un espace au sein duquel peuvent politiser leur expérience des individus qui ne cadrent pas avec un système pluraliste constitué de catégories identitaires discrètes, tels des personnes homosexuelles ou travesties et issues de la communauté africaine américaine ou chicana ${ }^{59}$.

31 Loin d'être un handicap, ce relatif manque d'unité identitaire permet que des personnes aux points de vue assez divers puissent s'employer à réaliser en commun un objectif restreint, car elles s'engagent mutuellement à respecter leurs différences, dans les limites qu'autorisent les besoins de cette cause. L'un des militants de ce collectif déclare :

Clearly we don't have Republicans and right-wingers working in this group, but there's a pretty wide range of left and progressive politics that might ordinarily be having strong debates about various qualms ${ }^{60}$.

Transatlantica, 1 | 2005 

donnent à ses participants une possibilité de réserve qui, loin d'affaiblir son efficacité, renforce sa capacité à asseoir sa légitimité sur une participation aussi diverse que possible. En effet, ce même militant décrit encore le collectif comme :

an informal setting where people do not make a commitment to an organization and everything the organization is doing, and where what these people are doing outside of this specific work is not regarded as reflecting on the work of the group ${ }^{61}$.

33 Ce collectif n'entreprend donc pas de mettre en pratique une idéologie spécifique d'ensemble, ce qui laisse à ses participants la possibilité de poursuivre, par ailleurs, une variété d'objectifs qui leur sont propres.

Mais, pour autant, les différences entre les participants ne sont aucunement gommées et leur action commune n'a rien de neutre (color-blind) en termes d'identités. Bien au contraire, l'identité des acteurs est tout sauf indifférente, puisqu'ils prennent part à l'action collective en tant qu'homosexuels, Noirs, femmes, Chicanos, travestis, Chinois ou prostitués ${ }^{62}$, par exemple : c'est, en effet, en tant que tel que l'on est plus fortement exposé au risque de subir des brutalités policières, qui touchent de manière disproportionnée les membres des minorités ethniques et sexuelles ${ }^{63}$. Il leur est cependant indispensable de s'allier, car, pris isolément, ni les personnes homosexuelles, ni les Noirs, ni les femmes, ni les Chicanos, ni les travestis, ni les Chinois, ni les prostitués ne seraient en mesure de se mesurer politiquement à la police. L'alliance est donc stratégique et ne vise en aucun cas à abolir les différences entre les partenaires, mais à les reconnaître et à les honorer, de façon à ce qu'elles constituent, non pas un obstacle à l'action collective, mais un facteur adjuvant. Ce collectif mobilise donc une identité collective polychrome, qui représente une véritable alternative à une gestion des identités par la couleur unie, qu'elle soit monochrome (une seule couleur) ou multicolore (plusieurs couleurs juxtaposées, mais chacune unie) et ce, d'autant plus que les catégories identitaires citées plus haut sont inclusives et susceptibles de se recouper. Il en résulte un continuum qui se rapproche effectivement du véritable spectre résultant de la réfraction de la lumière.

Ce type de configuration identitaire invalide l'idée selon laquelle tous les gais et les lesbiennes seraient appelés à former un groupe homogène et distinct, en vertu d'une hypothétique identité intrinsèque : «There is no essence of being queer that everybody shares whether they're a mass-murderer, a Republican, the head of Hitler's brown shirts, or a leftist » déclare ce même militant ${ }^{64}$. En choisissant des exemples aussi tranchés, voire caricaturaux, il se porte résolument en faux contre une conception communautaire largement répandue, qui dépolitise l'identité gaie et lesbienne en l'homogénéisant. Si son point de vue est représentatif de l'utilisation politique des identités par le collectif san-franciscain contre les brutalités policières, celui-ci repolitise donc l'homosexualité en mettant l'accent sur la mobilisation d'identités subjectives d'acteurs qui perçoivent leur homosexualité comme indissociable de convictions politiques progressistes et d'un désir de faire changer la société. En l'occurrence, l'action de ce collectif touche à une valeur capitale : le rôle de la police dans la société. Sa mission ne se limite donc pas, loin s'en faut, à un objectif de pure représentation d'intérêts particuliers, ni à la simple revendication d'obtenir justice d'incidents ponctuels. Son but est plutôt de porter à la connaissance du public des comportements policiers contraires à la mission de cette institution, de manière à la 
forcer à être pleinement responsable vis-à-vis de la société, ce qui implique une remise en cause de certains rapports de force sociaux.

Un tel dispositif ravive donc la dimension politique de l'identité gaie et lesbienne, en s'abstenant justement de la présupposer, et met en œuvre une forme de mobilisation gaie et lesbienne où cette identité n'est ni exclusive, ni insignifiante. Cet exemple illustre comment peut se pratiquer une réfraction qui disperse l'identité collective gaie et lesbienne en un spectre diffus et ravive ainsi une mobilisation où cette identité puisse tendre vers une véritable transformation de la société. Il s'agit d'une mobilisation identitaire bien plus offensive et audacieuse que celle qui se limite à obtenir le droit de participer à la vie publique à raison du poids propre à une communauté donnée.

La figure de l'arc-en-ciel permet de poser conjointement deux problèmes, distincts mais complémentaires, que sont, d'une part, le manque de prise en compte de la diversité ethnique et culturelle par la partie la plus visible du mouvement gai et lesbien et, d'autre part, la marchandisation de l'identité collective. Ces problèmes s'inscrivent dans une problématique de la continuité et de la discrétion des identités mises en œuvre dans la mobilisation homosexuelle. Tandis que le drapeau arc-en-ciel juxtapose des couleurs distinctes, l'arc-en-ciel est, lui, un dégradé continu ; de même, les identités sociales institutionnalisées sont fixes et monochromes, contrairement aux identités sociales vécues, qui sont fluides et polychromes.

La figure de l'arc-en-ciel aide donc à poser la question de la représentation et des déformations qu'elle peut occasionner dans une perspective qui est celle de l'authenticité65. Homogénéisation et marchandisation sont, en effet, deux modes d'instrumentalisation de l'identité gaie et lesbienne qui ont en commun de résulter d'un recours à l'identité dont l'initiative est prise, non pas par la base, mais par des leaders auxquels elle est déléguée. Ce sont inversement des initiatives partant de la base qui permettent de mobiliser une identité collective polychrome, c'est-à-dire qui échappe à deux travers qui ne sont contradictoires qu'en apparence : l'uniformisation et la segmentation. Le principe d'une authentique réfraction des identités collectives permet donc de dégager la pratique, aussi bien que l'étude, de mouvements sociaux identitaires de la dichotomie contraignante et réductrice universalisme - particularisme.

\section{BIBLIOGRAPHIE}

Ouvrages

AMNESTY INTERNATIONAL, Le Paradoxe américain (Paris : Les Editions Francophones d'Amnesty International, 1998).

ARMSTRONG, Elizabeth, Forging Gay Identities : Organizing Sexuality in San Francisco 1950-1994 (Chicago : University of Chicago Press, 2002). 
CASTELLS, Manuel, The City and the Grassroots : A Cross-Cultural Theory of Urban Social Movements (Berkeley : University of California Press, 1983).

CHASIN, Alexandra, Selling Out : The Gay and Lesbian Movement Goes to Market (Basingstoke : Palgrave MacMillan, 2000).

D'EMILIO, John, Sexual Politics, Sexual Communities. The Making of a Homosexual Minority in the United States : 1940-1970 (Chicago : University of Chicago Press, 1998 [1983]).

DUBERMAN, Martin, Stonewall (New York : Dutton, 1993).

ноокs, Bell, Feminist Theory : From Margin to Center (Boston : South End Press, 1984).

LUKENBILL, Grant, Untold Millions : Secret Truths about Marketing to Gay and Lesbian Consumers

(Binghamton, NY : Haworth, 1999).

MAROTTA, Toby, The Politics of Homosexuality (Boston : Houghton Mifflin, 1981).

MILLER, Neil, Out of the Past : Gay and Lesbian History from 1869 to the Present (New York : Vintage, 1995).

MORAGA, Cherríe et Gloria ANZALDÚA (dir.), This Bridge Called My Back : Writings by Radical Women of Color (Latham, NY : Kitchen Table, 1983).

PHELAN, Shane, Identity Politics. Lesbian Feminism and the Limits of Community (Philadelphie : Temple University Press, 1989).

REY-DEBOVE, Josette et Alain REY (dir.), Le Nouveau petit Robert (Paris : Dictionnaires Le Robert, 1993). STEIN, Arlene, Sex and Sensibility. Stories of a Lesbian Generation (Berkeley : University of California Press, 1997).

TAYLOR, Charles, The Malaise of Modernity (Concord, ONT : House of Anansi Press, 1991). [édition américaine : The Ethics of Authenticity (Cambridge : Harvard University Press, 1991)]

VAID, Urvashi, Virtual Equality : The Mainstreaming of Gay and Lesbian Liberation (New York : Doubleday, 1995).

\section{Articles}

ALONSO, Ana Maria et Maria Teresa KORECK, « Silences : "Hispanics”, AIDS, and Sexual Practices », in Henry ABELOVE, Michèle Aina BARALE et David HALPERIN (dir.), The Lesbian and Gay Studies Reader (New York : Routledge, 1993), 110-126.

AMALGUER, Tomás, « Chicano Men : A Cartography of Homosexual Identity and Behavior », in Henry ABELOVE et al. (dir.), The Lesbian and Gay Studies Reader, 255-273.

BADGET, M. V. Lee, « Beyond Biased Samples. Challenging the Myths on the Economic Status of Lesbians and Gay Men », in Amy GLUCKMAN et Betsy REED (dir.), Homo Economics : Capitalism, Community, and Lesbian and Gay Life (New York : Routledge, 1997), 65-71.

BERNSTEIN, Mary, «Celebration and Suppression : The Strategic Uses of Identity by the Lesbian and Gay Movement », American Journal of Sociology vol. 103, n 3 (novembre 1997), 531-565.

BLASIUS, Mark, « An Ethos of Lesbian and Gay Existence », Political Theory vol. 20, n 4 (novembre 1992), 642-671.

CASS, Vivienne, « Homosexual Identity : A Concept in Need of Definition », Journal of Homosexuality vol. 9, n 2/3 (1984), 105-126. 
COHEN, Cathy, « Contested Membership : Black Gay Identities and the Politics of AIDS ", in Steven SEIDMAN (dir.), Queer Theory/Sociology (Oxford : Oxford University Press, 1996), 362-394.

D’EMILIO, John, « Capitalism and Gay Identity », in Ann SNITOW, Christine STANSELL et Sharon THOMPSON (dir.), Powers of Desire: The Politics of Sexuality (Londres : Virago, 1984), 140-152.

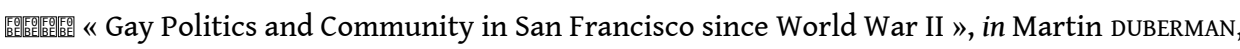
Martha VICINUS et George CHAUNCEY (dir.), Hidden from History : Reclaiming the Gay and Lesbian Past (New York, Meridian, 1990), 456-473.

EPSTEIN, Steven, « Gay Politics, Ethnic Identity : The Limits of Social Constructionism », Socialist Review vol. 17, n 3/4 (mai-août 1987), 9-54.

ESCOFFIER, Jeffrey, « Sexual Revolution and the Politics of Gay Identity », Socialist Review vol. 15, $\mathrm{n}^{\circ} 4 / 5$ (juillet-octobre 1985), 119-153.

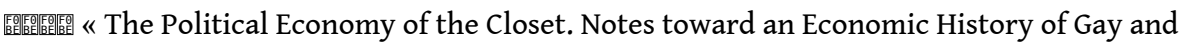
Lesbian Life before Stonewall ", in Amy GLUCKMAN et Betsy REED (dir.), Homo Economics : Capitalism, Community, and Lesbian and Gay Life, 123-134.

GLUCKMAN, Amy et Betsy REED, « The Gay Marketing Moment », in Amy GLUCKMAN et Betsy REED (dir.), Homo Economics : Capitalism, Community, and Lesbian and Gay Life, 3-9

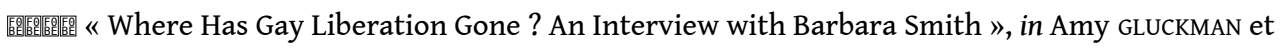
Betsy REED (dir.), Homo Economics : Capitalism, Community, and Lesbian and Gay Life, 195-207.

HOLLIBAUGH, Amber, « Seducing Women into "a Lifestyle of Vaginal Fisting" : Lesbian Sex Gets Virtually Dangerous ", in DANGERous BEDFELlows (dir.), Policing Public Sex : Queer Politics and the Future of AIDS Activism (Boston : South End Press, 1996), 321-336.

HUNTER, Nan, «Life after Hardwick », in Lisa DUGGAN et Nan HUNTER, Sex Wars : Sexual Dissent and Political Culture (New York, Routledge, 1995), 85-100.

MARCHE, Guillaume, « Homosexualité et ethnicité : rupture et recomposition d'un modèle identitaire », in Michel PRUM (dir.), La Peau de l'autre (Paris, Syllepse, 2001), 109-129.

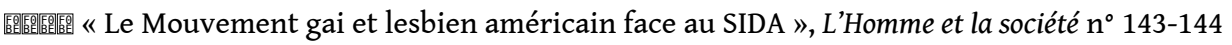
(janvier-juin 2002), 185-201.

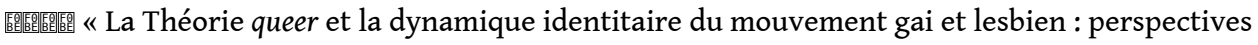
sociologiques sur un aspect des cultural studies ", in André KAENEL, Catherine LEJEUNE et Marie-Jeanne Rossignol (dir.), Cultural studies. Etudes culturelles (Nancy, Presses Universitaires de Nancy, 2003), 35-53.

MATTHAEI, Julie, « The Sexual Division of Labor, Sexuality, and Lesbian/Gay Liberation. Toward a Marxist-Feminist Analysis of Sexuality in U.S. Capitalism », in Amy GLUCKMAN et Betsy REED (dir.), Homo Economics : Capitalism, Community, and Lesbian and Gay Life, 135-164.

Pendleton, Eva, « Domesticating Partnerships », in DANGERous Bedfellows (dir.), Policing Public Sex, 373-393.

PEndeton, Eva et Jane GoldschmidT, « Sex Panic ! - Make the Connections », The Harvard Gay and Lesbian Review vol. 5, n 2 (été 1998), 30-33.

POLIKOFF, Nancy, « We Will Get What We Ask for : Why Legalizing Gay and Lesbian Marriage Will not "Dismantle the Legal Structure of Gender in Every Marriage" ", Virginia Law Review vol. 79, n 7 (octobre 1993), 1535-1550. 
QUIMBY, Ernest et Samuel FRIEDMAN, « Dynamics of Black Mobilization against AIDS in New York City », Social Problems vol. 36, nº 4 (1989), 403-415.

RICHARDSON, Diane, « Constructing Lesbian Sexualities », in Kenneth PLUMMER (dir.), Modern Homosexualities : Fragments of Lesbian and Gay Experience (New York : Routledge, 1992), 187-199.

SCHNEIDER, Beth, « Lesbian Politics and AIDS Work », in Kenneth PLUMMER (dir.), Modern Homosexualities, 160-174.

STEIN, Arlene, « The Year of the Lustful Lesbian », in Arlene STEIN (dir.), Sisters, Sexperts, Queers : Beyond the Lesbian Nation (New York : Penguin, 1993), 33-55.

YOUNG, Allen, « Out of the Closets, Into the Streets », in Karla JAY et Allen YounG (dir.), Out of the Closets : Voices of Gay Liberation (Londres : Gay Men's Press, 1992 [1972]), 6-31.

Presse et documents en ligne

ANDERSON, Steven, « The History of the Rainbow Flag », GAZE Magazine (Minneapolis) n 191, 28 mai 1993, p. 25 ; disponible sur PLANETOUT : http ://www.planetout.com/news/history/archive/ flag.html [15 octobre 2004].

BIG IDEA (THE), «Vision for an Economy Based on Co-operatives, Putting People before Profits », http ://www.emmett.ca/bigidea/ [16 novembre 2004].

GAY SHAME, « Gay Shame Opposes Marriage in any Form », http ://www.gayshamesf.org/info.htm [15 octobre 2004].

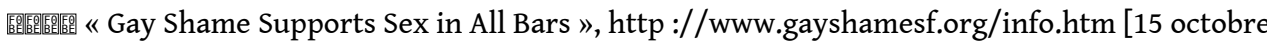
2004].

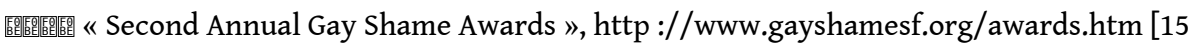
octobre 2004].

HERSCHER, Elaine, « Castro’s Change : Anti-Begging Drive Splits Area Known for Tolerance », San Francisco Chronicle, 12 mai 1998, sec. A, p. 17.

INTERNATIONAL COOPERATIVE ALLIANCE (ICA), « The History and Origins of the Rainbow Flag and International Co-operative Day », http ://www.coop.org/kids/rainbow.html [9 mai 2004].

JoHnson, Clarence, « Protesters Denounce S.F. Police Brutality », The San Francisco Chronicle, 12 juillet 1995, sec. A, p. 11.

LESBIAN AND GAY INSURRECTION (LAGAI), « Divorce Frenzy Sweeps Queer Nation : Clinton, Dole Unite to Stop It », Ultra-Violet vol. 7, nº 1 (juillet-août 1996), p. 1.

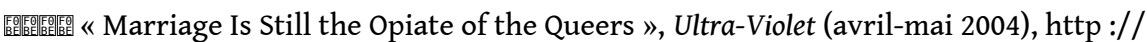
www.lagai.org/ [23 novembre 2004].

NIELSEN, Kate, « Lesbian Chic, Part Deux », The Advocate, 17 février 2004, http :// www.advocate.com/html/stories/908/908_nielsen.asp [24 janvier 2005].

Q GIFTSHOP (page d'accueil), http ://www.qgiftsonline.com/ [26 octobre 2004].

RAINBOW GIFTS (page d'accueil), http ://rainbowgifts.zoovy.com/ [26 octobre 2004].

SCHWARTZ, Stephen, « S.F. Group Protests Raid of AIDS Benefit », The San Francisco Chronicle, 12 janvier 1995, sec. A, p. 19.

SWARD, Susan, Bill WALLACE et Maitland ZANE, «Police Beating », The San Francisco Chronicle, 6 juin 1995, sec. A, p. 1. 
UNCLE DONALD'S CASTRO STREET, « Harvey Milk 20 Years After », 2 juin 2003, http ://thecastro.net/ milk/milk+20.html [26 octobre 2004].

WIELENGA, Dave, «Is That a Badge in Your Pocket? Or are you just happy to see me? Are OC's cops a little too excited about busting men for gay sex? ", Orange County Weekly, vol. 4, n² 28, 19-25 mars 1999, http ://www.ocweekly.com/ink/archives/99/28lede-wielenga.shtml [26 novembre 2004].

\section{NOTES}

1. Depuis le début des années 1990, cette communauté se désigne elle-même habituellement comme « gaie, lesbienne, bisexuelle et trans-genre » (GLBT ou LGBT) suite à une forte revendication d'inclusion de la part de personnes ne se reconnaissant pas pleinement dans une identité collective définie uniquement en termes d'homosexualité. Voir notamment : Loraine HUTCHINS et Lani KA'AHUMANU (dir.), By Any Other Name : Bisexual People Speak Out (Boston : Alyson, 1991) ; Naomi TUCKER (dir.), Bisexual Politics : Theories, Queries, and Visions (New York, Haworth, 1995). Souvent, cependant, le changement de terminologie reflète davantage une volonté d'affirmer que les personnes bisexuelles et trans-genre font également partie de cette communauté qu'une profonde remise en cause de l'identité collective autour de laquelle elle rassemble, si bien que l'inclusion des termes «bisexuel » et « trans-genre » dans le label identitaire témoigne plutôt, en réalité, de la neutralisation de la remise en cause représentée par ces deux dimensions de la déviance sexuelle par la dynamique communautaire étudiée ici. Voir : Elizabeth ARMSTRONG, Forging Gay Identities : Organizing Sexuality in San Francisco 1950-1994 (Chicago, University of Chicago Press, 2002), 178-184. C'est pourquoi nous faisons ici le choix de nous concentrer sur la dimension homosexuelle de cette communauté, afin de rendre compte du fait que le paradigme de l'homosexualité reste dominant dans l'identification et la mobilisation collectives de cette communauté, malgré les nombreux débats, remises en cause et questionnements dont l'ampleur dépasse le cadre de cette étude. De fait, la rhétorique queer de remise en cause systématique des catégories identitaires, en particulier, ne constitue pas un paradigme opératoire dans les pratiques des acteurs, même si elle est riche d'enseignements quant au caractère construit des identités. Voir : Guillaume MARCHE, « La Théorie queer et la dynamique identitaire du mouvement gai et lesbien : perspectives sociologiques sur un aspect des cultural studies ", in André KAENEL, Catherine LEJEUNE et Marie-Jeanne ROSSIGNOL (dir.), Cultural studies. Etudes culturelles (Nancy, Presses Universitaires de Nancy, 2003), 35-53. Dans notre choix de focaliser cet article sur la dimension homosexuelle de son objet, il ne faut donc pas lire la validation d'une conception exclusivement binaire de la sexualité (hétéro-homo), du genre (masculin-féminin) et du sexe (homme-femme) ; il s'agit plutôt, en l'occurrence, de faire honneur à la question de la bisexualité et du franchissement des frontières de genre (recouvrant notamment le travestissement et le transsexualisme), en ne faisant pas des défis qu'elle représente une dimension annexe des problèmes étudiés ici. 2. Nous n'entrerons pas ici dans les implications conceptuelles - plutôt essentialistes ou au contraire plutôt constructivistesdu choix de l'une ou l'autre expression. 3. INTERNATIONAL COOPERATIVE ALLIANCE (ICA), «The History and Origins of the Rainbow Flag and International Co-operative Day », http ://www.coop.org/kids/rainbow.html [9 
mai 2004] ; THE BIG IDEA, « Vision for an Economy Based on Co-operatives, Putting People before Profits ", http ://www.emmett.ca/bigidea/ [16 novembre 2004].

4. Steven ANDERSON, «The History of the Rainbow Flag », GAZE Magazine (Minneapolis) n ${ }^{\circ}$ 191, 28 mai 1993, p. 25 ; disponible sur PLANETOUT : http ://www.planetout.com/news/ history/archive/flag.html [15 octobre 2004].

5. Neil MILLER, Out of the Past : Gay and Lesbian History from 1869 to the Present (New York : Vintage, 1995), 395-411; John D’EMILIO, « Gay Politics and Community in San Francisco since World War II », in Martin DUBERMAN, Martha vicinus et George CHAUnCEY (dir.), Hidden from History : Reclaiming the Gay and Lesbian Past (New York : Meridian, 1990), 468-473.

6. Jusqu'à la fin des années 1970 , le terme "gay » désignait indistinctement des hommes ou des femmes ayant décidé de vivre leur homosexualité au grand jour ; c'est le mouvement féministe lesbien (lesbian feminist) qui permit une prise de conscience progressive du fait que «gay » correspondait dans les faits à une identité surtout masculine. L'usage conjoint des termes « gay and lesbian », ou « lesbian and gay » ne s'est répandu qu'à partir des années 1980 , lorsque le sida occasionna une nouvelle alliance stratégique entre lesbiennes et gais (voir plus bas).

7. Allen YounG, « Out of the Closets, Into the Streets ", in Karla JAY et Allen Young (dir.), Out of the Closets : Voices of Gay Liberation (Londres : Gay Men's Press, 1992 [1972]), 6-31 ; Mark BLASIUS, « An Ethos of Lesbian and Gay Existence ", Political Theory vol. 20, $\mathrm{n}^{\circ} 4$ (novembre 1992), 654-660.

8. Manuel CASTELLS, The City and the Grassroots : A Cross-Cultural Theory of Urban Social Movements (Berkeley : University of California Press, 1983), 140-166.

9. John D’EMILIO, « Gay Politics and Community », 466-468; Jeffrey ESCOFFIER, « The Political Economy of the Closet. Notes toward an Economic History of Gay and Lesbian Life before Stonewall », in Amy GLUCKMAN et Betsy REED (dir.), Homo Economics: Capitalism, Community, and Lesbian and Gay Life (New York : Routledge, 1997), 127-131.

10. John D'EMILIO, «Capitalism and Gay Identity ", in Ann SNITOW, Christine STANSELl et Sharon THомPSON (dir.), Powers of Desire: The Politics of Sexuality (Londres : Virago, 1984), 140-152.

11. Arlene STEIN, Sex and Sensibility. Stories of a Lesbian Generation (Berkeley : University of California Press, 1997), 35-46 et 112-120.

12. Shane PHELAN, Identity Politics. Lesbian Feminism and the Limits of Community (Philadelphie, Temple University Press, 1989), 47-52 et 69-76.

13. Manuel CASTELLS, The City and the Grassroots, 163-170.

14. Voir : UNCLE DONALD'S CASTRO STREET, « Harvey Milk 20 Years After », 2 juin 2003, http ://thecastro.net/milk/milk+20.html [26 octobre 2004].

15. John D'EmiLio, Sexual Politics, Sexual Communities. The Making of a Homosexual Minority in the United States : 1940-1970 (Chicago : University of Chicago Press, 1998 [1983]),

231-233 ; voir aussi : Martin DUBERMAN, Stonewall (New York : Dutton, 1993), 219-233 ; Toby MAROTTA, The Politics of Homosexuality (Boston : Houghton Mifflin, 1981), ch. 4, 6 et 8.

16. Guillaume MARCHE, «Le Mouvement gai et lesbien américain face au SIDA », L'Homme et la société, $\mathrm{n}^{\circ}$ 143-144 (janvier-juin 2002), 186-188.

17. Elizabeth ARMSTRONG, Forging Gay Identities, 68-72.

18. Jeffrey ESCOFFIER, « The Political Economy of the Closet », 130-131. 
19. Voir par exemple : $Q$ GIFTSHOP (page d'accueil), http ://www.qgiftsonline.com/; RAINBOW GIFTS (page d'accueil), http ://rainbowgifts.zoovy.com/ [26 octobre 2004].

20. Entretien avec un militant de longue date du mouvement gai et lesbien, impliqué en particulier dans la lutte contre l'exclusion socio-économique qui résulte de l'embourgeoisement (gentrification) du quartier du Castro, San Francisco, 15 juillet 1998 ; Elaine HERSCHER, « Castro's Change : Anti-Begging Drive Splits Area Known for Tolerance », San Francisco Chronicle, 12 mai 1998, sec. A, p. 17.

21. Entretien avec un jeune militant gai, impliqué notamment dans l'amélioration des relations entre les populations portoricaine et homosexuelle du quartier de South End, Boston, 5 août 1997.

22. Steven EPSTEIN, « Gay Politics, Ethnic Identity : The Limits of Social Constructionism », Socialist Review vol. 17, n 3/4 (mai-août 1987), 17-23.

23. Elizabeth ARMSTRONG, Forging Gay Identities, 102-110.

24. Mary BERNSTEIN, «Celebration and Suppression : The Strategic Uses of Identity by the Lesbian and Gay Movement ", American Journal of Sociology vol. 103, $n^{\circ} 3$ (novembre 1997), 542-549.

25. Jeffrey ESCOFFIER, « Sexual Revolution and the Politics of Gay Identity », Socialist Review vol. 15, n $4 / 5$ (juillet-octobre 1985), 141-145; Guillaume MARCHE, « Homosexualité et ethnicité : rupture et recomposition d'un modèle identitaire », in Michel PRUM (dir.), La Peau de l'autre (Paris : Syllepse, 2001), 114-118.

26. Vivienne CASS, « Homosexual Identity : A Concept in Need of Definition ", Journal of Homosexuality vol. 9, n 2/3 (1984), 118-121; Steven EPSTEIN, « Gay Politics, Ethnic Identity ", 34-42.

27. Elizabeth ARMSTRONG, Forging Gay Identities, 147-153 ; Guillaume MARCHE, « Homosexualité et ethnicité », 118-124.

28. Conformément à un usage désormais établi en français, nous orthographions sida en minuscules.

29. Nan HUNTER, «Life after Hardwick », in Lisa DUGGAN et Nan HUNTER, Sex Wars : Sexual Dissent and Political Culture (New York : Routledge, 1995), 85-100.

30. Notons que cette inflexion de la stratégie de défense des droits des gais et des lesbiennes eut pour effet de renforcer la désexualisation des représentations du lesbianisme, alors même que le sida avait été l'occasion d'un fort engagement de la part de lesbiennes désireuses, loin de subordonner leur identité collective à celle des hommes gais, de faire valoir leurs propres revendications en tant que lesbiennes, en portant sur le devant de la scène des formulations identitaires alternatives au féminisme lesbien, mettant notamment en exergue une affirmation offensive du caractère sexuel de leur lesbianisme, à l'aide de slogans tels que «dykes fuck, too ». Voir : Diane RICHARDSON, «Constructing Lesbian Sexualities », in Kenneth PLUMMER (dir.), Modern Homosexualities : Fragments of Lesbian and Gay Experience (New York : Routledge, 1992), 187-199 ; Beth SCHNEIDER, « Lesbian Politics and AIDS Work », in Kenneth PLUMMER (dir.), Modern Homosexualities, 160-174.

31. Tomás AMALGUER, « Chicano Men : A Cartography of Homosexual Identity and Behavior ", in Henry ABELOVE et al. (dir.), The Lesbian and Gay Studies Reader (New York: Routledge, 1993), 257-262 ; Ana Maria ALONSO et Maria Teresa KORECK, « Silences : "Hispanics", AIDS, and Sexual Practices ", in Henry ABELove et al. (dir.) The Lesbian and Gay Studies Reader, 115-119; Ernest QUIMBY et Samuel FRIEDMAN, « Dynamics of Black Mobilization against AIDS in New York City ", Social Problems vol. 36, $\mathrm{n}^{\circ} 4$ (1989), 
405-407 ; Cathy COHEN, « Contested Membership : Black Gay Identities and the Politics of AIDS », in Steven SEIDMAN (dir.), Queer Theory/Sociology (Oxford : Oxford University Press, 1996), 378-382.

32. Voir notamment : Cherríe MORAGA et Gloria ANZALDÚA (dir.), This Bridge Called My Back: Writings by Radical Women of Color (Latham, NY : Kitchen Table, 1983) ; Bell HоокS, Feminist Theory : From Margin to Center (Boston : South End Press, 1984).

33. Entretien avec un responsable de l'association d'Africains Américains gais Gay Men of African Descent (GMAD), New York, 19 août 1998 ; entretien avec un Latino ancien membre d'ACT UP, San Francisco, 23 juillet 1998.

34. Guillaume MARCHE, « Homosexualité et ethnicité », 120-124.

35. Amy GLUCKMAN et Betsy REED, " The Gay Marketing Moment », in Amy GLUCKMAN et Betsy REED (dir.), Homo Economics, 3-9 ; voir aussi : Alexandra CHASIN, Selling Out : The Gay and Lesbian Movement Goes to Market (Basingstoke : Palgrave MacMillan, 2000) ; Grant LUKENBILL, Untold Millions : Secret Truths about Marketing to Gay and Lesbian Consumers (Binghamton, NY : Haworth, 1999).

36. M. V. Lee BADGET, «Beyond Biased Samples. Challenging the Myths on the Economic Status of Lesbians and Gay Men », in Amy GLUCKMAN et Betsy REED (dir.), Homo Economics, 65-71; voir aussi : Julie MATTHAEI, « The Sexual Division of Labor, Sexuality, and Lesbian/Gay Liberation. Toward a Marxist-Feminist Analysis of Sexuality in U.S. Capitalism », in Amy GLUCKMAN et Betsy REED (dir.), Homo Economics, p. 135-164..

37. En 1993, le magazine grand public New York Magazine fit l'une de ses couvertures sur le thème "Lesbian Chic », tandis que, la même année, Vanity Fair et Newsweek utilisaient la notoriété de chanteuses ouvertement lesbiennes telles que k. d. lang et Melissa Etheridge pour réaliser des couvertures accrocheuses sur le thème de ces lesbiennes d'un genre supposé nouveau. Voir : Kate NIELSEN :« Lesbian Chic, Part Deux », The Advocate, 17 février 2004, http ://www.advocate.com/html/stories/908/908_nielsen.asp [24 janvier 2005]; Urvashi vAID, Virtual Equality: The Mainstreaming of Gay and Lesbian Liberation (New York: Doubleday, 1995), 80-81.

38. Josette REY-DEBOVE et Alain REY (dir.), Le Nouveau petit Robert (Paris : Dictionnaires Le Robert, 1993), p. 129.

39. Guillaume MARCHE, «Le Mouvement gai et lesbien américain face au SIDA », 189-194.

40. Urvashi VAID, Virtual Equality, ch. 7 et 8 ; Amy GLUCKMAN et Betsy REED, « Where Has Gay Liberation Gone? An Interview with Barbara Smith », in Amy GLUCKMAN et Betsy REED (dir.), Homo Economics, 195-207.

41. GAY SHAME, « Gay Shame Opposes Marriage in any Form », http :// www.gayshamesf.org/info.htm [15 octobre 2004].

42. Voir : Nancy POLIKOFF, « We Will Get What We Ask for : Why Legalizing Gay and Lesbian Marriage Will not "Dismantle the Legal Structure of Gender in Every Marriage" ", Virginia Law Review vol. 79, n 7 (octobre 1993), 1535-1550.

43. GAY SHAME, « Second Annual Gay Shame Awards », http ://www.gayshamesf.org/ awards.htm [15 octobre 2004].

44. Cette idée fait l'objet d'un démenti magistral de la part de M. V. Lee Badget dans «Beyond Biased Samples », 65-71. L'auteur y explique, d'une part, que les statistiques visant à démontrer que le pouvoir d'achat de la population gaie et lesbienne est supérieur à la moyenne résultent souvent de sondages effectués auprès du lectorat de la presse commerciale gaie et lesbienne, qui, vu ses thèmes de prédilection et son prix 
de vente, s'adresse de facto à une tranche moyenne à aisée de cette population. D'autre part, pour mesurer l'impact effectif de l'orientation sexuelle des personnes sur leur revenu, il faut, en réalité, parvenir à neutraliser l'effet de l'ensemble des autres facteurs qui influent sur la rémunération, à savoir : le niveau d'études, l'âge, le lieu de résidence, l'origine ethnique et le sexe. Les résultats d'une telle étude, réalisée par $\mathrm{M}$. $\mathrm{V}$. Lee Badget, permettent de conclure que les gais comme les lesbiennes gagnent en fait moins que les hommes et les femmes hétérosexuels qui leur sont comparables. $\mathrm{Si}$, considérant l'ensemble de la population, le revenu moyen des couples hétérosexuels est moindre que celui des couples homosexuels, c'est, selon cette étude, parce que davantage d'hommes que de femmes s'identifient publiquement comme homosexuels, de sorte que les couples gais identifiables à des fins statistiques sont plus nombreux que les couples lesbiens. La différence de revenu entre les couples homosexuels et hétérosexuels n'est donc pas imputable à une quelconque discrimination à rebours à l'avantage des gais ou des lesbiennes, mais à la discrimination économique qui s'exerce à l'encontre des femmes.

45. Notons que, outre le fait qu'elles contribuent à la marchandisation de l'identité collective gaie et lesbienne étudiée ici, de telles représentations sont fréquemment utilisées pour justifier le refus de certains responsables politiques et juges conservateurs d'accorder une protection contre la discrimination en fonction de l'orientation sexuelle, au motif que, loin d'être victimes d'injustice, les homosexuels - l'attention se portant prioritairement sur les hommes - constitueraient une population objectivement privilégiée.

46. LAGAI, « Marriage Is Still the Opiate of the Queers », Ultra-Violet (avril-mai 2004), http ://www.lagai.org/ [23 novembre 2004].

47. Voir Allen Young, « Out of the Closets, Into the Streets », 28-31.

48. LAGAI, «Divorce Frenzy Sweeps Queer Nation : Clinton, Dole Unite to Stop It », Ultra-Violet vol. 7, n 1 (juillet-août 1996), p. 1.

49. Le Defense of Marriage Act (DOMA) est une loi définissant, au regard de l'Etat fédéral, le mariage comme l'union d'un homme et d'une femme et autorisant de manière préventive tout Etat à ne pas reconnaître un mariage homosexuel conclu dans un autre Etat.

50. Entretien avec une responsable de LaGAI, San Francisco, 22 juillet 1998.

51. Entretien avec une responsable de LaGAI, San Francisco, 22 juillet 1998 ; LAGAI, «Marriage Is Still the Opiate of the Queers".

52. Dave WIELENGA, «Is That a Badge in Your Pocket? Or are you just happy to see me? Are OC's cops a little too excited about busting men for gay sex ? ", Orange County Weekly, vol. 4, n² 28, 19-25 mars 1999, http ://www.ocweekly.com/ink/archives/ 99/28lede-wielenga.shtml [26 novembre 2004] ; GAY SHAME, « Gay Shame Supports Sex in All Bars », http ://www.gayshamesf.org/info.htm [15 octobre 2004].

53. Eva PENDLETON et Jane GOLDSCHMIDT, «Sex Panic! - Make the Connections », The Harvard Gay and Lesbian Review vol. 5, n 2 (été 1998), 30-33.

54. Eva PENDLETON, « Domesticating Partnerships », in DANGERous BEDFELLows (dir.), Policing Public Sex : Queer Politics and the Future of AIDS Activism (Boston : South End Press, 1996), 373-393.

55. Arlene STEIN, « The Year of the Lustful Lesbian », in Arlene STEIN (dir.), Sisters, Sexperts, Queers : Beyond the Lesbian Nation (New York : Penguin, 1993), 33-55 ; Amber HOLLIBAUGH, « Seducing Women into "a Lifestyle of Vaginal Fisting" : Lesbian Sex Gets Virtually Dangerous ", in DANGERous BEDFELLows (dir.), Policing Public Sex, 321-336. 
56. Amber hollibaugh, « Seducing Women », 334-335.

57. Stephen SCHWARTZ, « S.F. Group Protests Raid of AIDS Benefit », The San Francisco Chronicle, 12 janvier 1995, sec. A, p. 19 ; Susan SWARD, Bill WALLACE et Maitland ZANE, « Police Beating ", The San Francisco Chronicle, 6 juin 1995, sec. A, p. 1.

58. Clarence JoHnson, "Protesters Denounce S.F. Police Brutality », The San Francisco Chronicle, 12 juillet 1995, sec. A, p. 11.

59. Entretien avec un militant de ce collectif de lutte contre les brutalités policières, San Francisco, 11 juillet 1998.

60. Entretien avec un militant de ce collectif, San Francisco, 11 juillet 1998.

61. Entretien avec un militant de ce collectif, San Francisco, 11 juillet 1998.

62. Précisons que, conformément à l'usage français, « ou » doit être compris de manière inclusive. C'est par souci de lisibilité de la phrase que nous employons ici le masculin au sens générique.

63. AMNESTY INTERNATIONAL, Le Paradoxe américain (Paris : Les Editions Francophones d'Amnesty International, 1998), 34-43 et 59-66.

64. Entretien, 11 juillet 1998.

65. Voir : Charles TAYLOR, The Malaise of Modernity (Concord, ONT : House of Anansi Press, 1991), 32-41. 